\title{
A new tool for diagnosing parathyroid lesions: angio plus ultrasound imaging
}

\author{
He Liu ${ }^{1 \#}$, Quan Liao ${ }^{2 \#}$, Yong Wang ${ }^{3 \#}$, Ya Hu${ }^{2}$, Qingli Zhu ${ }^{1}$, Liang Wang ${ }^{1}$, Qianqian Liu ${ }^{1}$ Jianchu Li ${ }^{1 *}$, \\ Yuxin Jiang ${ }^{1 *}$
}

${ }^{1}$ Department of Ultrasound, ${ }^{2}$ Department of Surgery, Peking Union Medical College Hospital, Chinese Academy of Medical Sciences \& Peking Union Medical College, Beijing 100730, China; ${ }^{3}$ Department of Ultrasound, Cancer Hospital Chinese Academy of Medical Sciences, Beijing 100021, China

Contributions: (I) Conception and design: H Liu, Q Liao, Y Wang, J Li, Y Jiang; (II) Administrative support: J Li, Y Jiang; (III) Provision of study materials or patients: Q Liao, Y Hu; (IV) Collection and assembly of data: H Liu, Y Wang, Q Zhu; (V) Data analysis and interpretation: H Liu, L Wang, Q Liu; (VI) Manuscript writing: All authors; (VII) Final approval of manuscript: All authors.

\#These authors contributed equally to this work.

*These authors contributed equally to this work.

Correspondence to: Jianchu Li; Yuxin Jiang. Department of Ultrasound, Peking Union Medical College Hospital, Chinese Academy of Medical

Sciences \& Peking Union Medical College, Shuaifuyuan 1, Wangfujing, Dongcheng District, Beijing 100730, China.

Email: lijianchuxhbj@126.com; jiangyuxinxhbj@126.com.

Background: Our aim was to examine the potential value of angio plus ultrasound imaging in diagnosing parathyroid lesions. Angio plus ultrasound imaging uses a new paradigm in Doppler performance, which allows for a better detection of flow in small vessels while maintaining workflow of conventional color flow imaging characteristics.

Methods: Thirty parathyroid lesions, composed of 26 histopathologically diagnosed adenoma and 4 hyperplasia (two hyperplasia in the same patient), from 29 consecutive patients (6 men and 23 women; median age: 53 years, range: $25-78$ years) were evaluated using both color Doppler and angio plus ultrasound imaging. The polar vessel (visible or invisible), number and distribution (peripheral, central, and both) of blood flow signals were compared between the two techniques.

Results: On color Doppler, the polar vessel was visible in $6(20 \%)$ lesions. The median number of blood flow signals was 6 [3-23]. The distribution of peripheral, central and both was shown in 4 (13.3\%), 9 (30\%), and $15(50 \%)$ lesions respectively, and no blood signals was shown in 2 (6.7\%) lesions. On angio plus ultrasound imaging, the polar vessel was visible in $16(53.3 \%)$ lesions. The median number of blood flow signals was 3 [0-18]. The distribution of peripheral, central and both was shown in 1 (3.3\%), 4 (13.3\%), and $25(83.3 \%)$ lesions respectively. There was significant difference between color Doppler and angio plus ultrasound imaging in the detection of the polar vessel, number and distribution of blood flow signals.

Conclusions: The typical polar vessel and increased vascularity commonly associated with parathyroid lesions may be obtained with angio plus ultrasound imaging. Angio plus ultrasound imaging can improve the detection rate and distribution characteristics of blood flow signals of parathyroid lesions. Further research is needed to clarify the clinical meaning of increased blood flow information, such as the differentiation of parathyroid lesions from other lesions.

Keywords: Hyperparathyroidism; ultrasound; angio plus; color Doppler

Submitted Sep 10, 2019. Accepted for publication Nov 12, 2019.

doi: $10.21037 /$ jtd.2019.11.29

View this article at: http://dx.doi.org/10.21037/jtd.2019.11.29

(C) Journal of Thoracic Disease. All rights reserved. 


\section{Introduction}

A wide variety of imaging techniques, including sestamibi based scintigraphy, ultrasound, CT, and magnetic resonance imaging have been used alone and in combination to localize parathyroid lesions. Ultrasound and technetium 99mesestamibi scintigraphy are usually the first line diagnostic modalities for routine preoperative imaging localization of a parathyroid lesions (1). However, sestamibi based scintigraphy has several limitations, including poor anatomic detail of sestamibi scans. In contrast, ultrasound provides good anatomic information about masses in the neck, which is the key to successful parathyroid surgery in the era of minimally invasive parathyroid surgery. Ultrasound has been used as the main imaging tool for preoperative localization of the hyperfunctioning parathyroid gland. Adding color and power Doppler to ultrasound exam improves detection of parathyroid lesions (2-4). Enlarged parathyroid glands are more hypervascular than nodular thyroid lesions and the detection of polar vessel (a feeding artery) is highly suggestive of a parathyroid lesion (5-9). Recently, angio plus ultrasound imaging has emerged as a complementary tool for the visualization of extremely low velocity flows that are typically removed on conventional color and power Doppler without significant motion artifacts. Angio plus ultrasound imaging uses a new paradigm in Doppler performance, which allows for a better detection of flow in small vessels while maintaining workflow of conventional color flow imaging characteristics. To date, no papers have been published on the use of angio plus ultrasound imaging in parathyroid lesions. Therefore, the purpose of this study was to demonstrate the early experience of angio plus ultrasound imaging in hyperparathyroidism patients by comparing it with conventional color Doppler in the detection of polar vessel, the number and distribution of blood flow signals.

\section{Methods}

\section{Patients}

The institutional review board of Peking Union Medical College Hospital approved the protocol (approval number: ZS-1267), and written informed consent was obtained from all patients before ultrasound examination. We carried out this prospective study from January 2018 to April 2018, enrolled 29 consecutive hyperparathyroidism patients scheduled for parathyroid surgery and with both positive neck ultrasound and MIBI scan. Surgical pathology results were considered as the reference standard (mean length of time between ultrasound and surgery $=3$ days, range $1-7$ days).

\section{Equipment and data acquisition}

All ultrasound examinations were performed by a radiologist with 15 years of experience in thyroid and neck ultrasound, as well as 15 months experience using angio plus ultrasound imaging before study. Supersonic Aixplorer ultrasound system (Supersonic Imagine, Provence, France) equipped with a15-4 MHz linear array transducer was used. Each patient was placed in a supine position with neck slightly extended and the transducer applied gently with minimal pressure.

Gray-scale images were obtained first, and then color Doppler and angio plus imaging were performed. Color Doppler was performed with standardized parameters to allow detection of low velocity blood flow: $4-6 \mathrm{~cm} /$ $\mathrm{s}$ velocity scale, low wall filter, as high gain as possible, medium dynamic range. The vascularity of the lesion was observed and recorded using color Doppler and angio plus imaging, respectively. All images and loops were stored for subsequent analysis. The combined examination time of grayscale, color Doppler, and angio plus imaging was between 10 and 15 minutes.

\section{Image analysis}

A radiologist ( $\mathrm{H} \mathrm{Liu)} \mathrm{performed} \mathrm{all} \mathrm{ultrasound} \mathrm{examinations}$ and stored the images in the machine for later analysis. Two radiologists ( $\mathrm{H} \mathrm{Liu} \mathrm{and} \mathrm{Q} \mathrm{Zhu)} \mathrm{were} \mathrm{blinded}$ to the patients' clinical data and analyzed the images independently to classify the blood flow characteristics. If disagreement occurred, two readers jointly reviewed the images, discussed and came to a consensus. The polar vessel, number and distribution of blood flow signals were assessed in reference to previous studies (10). Polar vessel was defined as the demonstration of any vessel enveloping 90 to 270 degrees of the parathyroid lesion (11). The number of distinct vessels was counted. The distribution was categorized as peripheral, central, or both. Vascular findings were compared between color Doppler and angio plus imaging.

\section{Statistical analysis}

Statistical analyses were performed using SPSS 18.0 Statistical Software for Windows. Demographic and clinical 

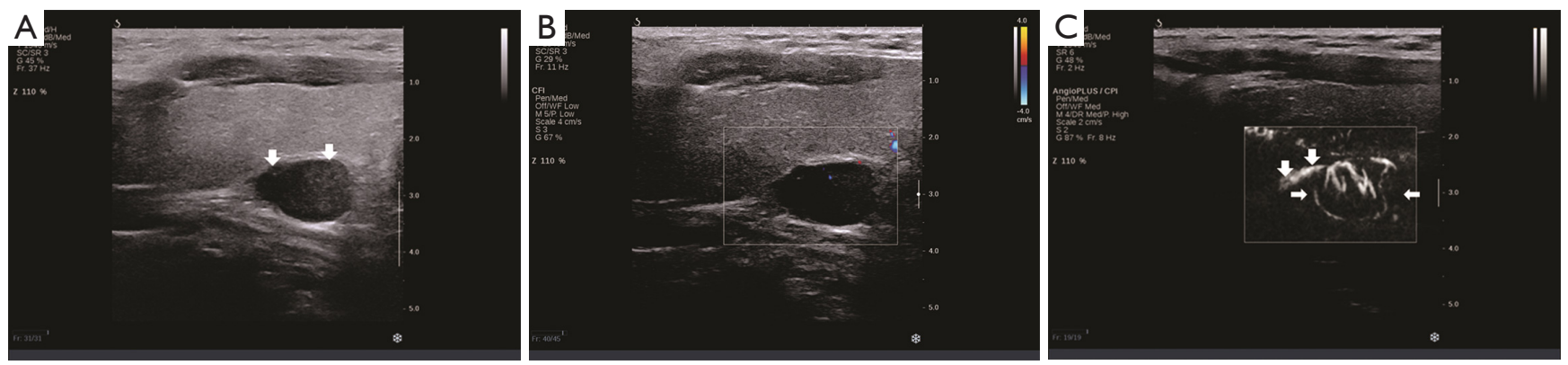

Figure $1 \mathrm{~A} 44$-year-old man with right superior parathyroid hyperplasia, $1.8 \mathrm{~cm} \times 1.5 \mathrm{~cm} \times 1.4 \mathrm{~cm}$ in size on longitudinal scan. It is seen as a hypoechoic lesion (arrows) on the posterior surface of the middle segment of the right thyroid lobe (A), with few blood flow signals on color Doppler (B). On angio plus ultrasound imaging, it showed the polar vessel (vertical arrows) and abundant blood flow signals (horizontal arrows) (C).

Table 1 the polar vessel detected by color Doppler and angio plus ultrasound imaging

\begin{tabular}{lccc}
\hline \multirow{2}{*}{ Variable } & \multicolumn{2}{c}{ Angio plus } & Total \\
\cline { 2 - 3 } & Presence of pole & Absence of pole & \\
\hline Color Doppler & 6 & 0 & 6 \\
Presence of pole & 10 & 14 & 24 \\
Absence of pole & 16 & 14 & 30 \\
Total & & & \\
\hline
\end{tabular}

characteristics are summarized as medians and ranges and $\mathrm{N}$ and percent, as appropriate. The number of blood flow signals detected by color Doppler and angio plus was compared by non-parametric Mann-Whitney $U$ test. Fisher's exact test and the $\chi^{2}$ test were used to analyze the difference in polar vessel and vascular distribution between the two imaging techniques. To evaluate the inter-observer agreement of vascular patterns, the intra-class correlation coefficient (ICC) and k-coefficient were calculated. For all tests, a $\mathrm{P}$ value $<0.05$ was considered statistically significant.

\section{Results}

Twenty-six histopathologically diagnosed parathyroid adenoma and 4 hyperplasia (two hyperplasia in the same patient) from 29 consecutive patients ( 6 men and 23 women; median age: 53 years, range: $25-78$ years) were included in our study. Among them, 19 adenomas and 1 hyperplasia originated from inferior parathyroid glands, 7 adenomas and 3 hyperplasia originated from superior parathyroid glands. The median maximum diameter of the parathyroid lesions was 1.9 (range: $0.9-4.0) \mathrm{cm}$.

About two more minutes were needed to acquire additional angio plus images for a patient. Angio plus ultrasound imaging revealed more vessels than color Doppler $(\mathrm{P}=0.002)$ (Figure $1 A, B, C)$. The median number of vessels detected by angio plus ultrasound imaging was 6 and that by color Doppler was 3. Two parathyroid lesions with no blood flow signal on color Doppler showed 3 blood vessels on angio plus ultrasound imaging. Eight parathyroid lesions with one or two blood vessels on color Doppler showed 3 or more than 3 blood vessels on angio plus ultrasound imaging. Angio plus ultrasound imaging performed better than color Doppler in displaying the polar vessel around the lesion (Table 1, $\mathrm{P}=0.019$ ) (Figure 1A,B,C). Angio plus ultrasound imaging displayed the polar vessel in 16 lesions (53.3\%), while color Doppler did so in $6(20.0 \%)$ lesions (Table 1). On angio plus ultrasound imaging, 25 $(83.3 \%)$ lesions demonstrated peripheral and central blood flow, $4(13.3 \%)$ lesions showed internal blood flow, and $1(3.3 \%)$ lesion had peripheral blood flow. In contrast, peripheral and central, internal, peripheral, and no blood flow was observed in 15 (50.0\%), 9 (30.0\%), 4 (13.3\%), 2 $(6.7 \%)$ lesions respectively (Table 2$)$. Significant difference existed in displaying the distribution of blood flow between these two techniques $(\mathrm{P}=0.007)$.

The inter-observer reproducibility acquired by 2 independent operators was assessed. For color Doppler, the ICC of the number of blood vessels was 0.702 ; the k-coefficient of vascular pole and distribution was 0.583 and 0.434 . For angio plus imaging, the intraclass correlation coefficient of the number of blood vessels was 0.760; the k-coefficient of vascular pole and distribution 
Table 2 the distribution of blood flow signals detected by color Doppler and angio plus ultrasound imaging

\begin{tabular}{lcccc}
\hline \multirow{2}{*}{ Variable } & \multicolumn{3}{c}{ Angio plus } & \multirow{2}{*}{ Total } \\
\cline { 2 - 3 } & Peripheral & Internal & Both & \\
\hline Color Doppler & 1 & 1 & 0 & 2 \\
None & 0 & 0 & 4 & 4 \\
Peripheral & 0 & 3 & 6 & 9 \\
Internal & 0 & 0 & 15 & 15 \\
Both & 1 & 4 & 25 & 30 \\
Total & & & & \\
\hline
\end{tabular}

was 0.529 and 0.500 .

\section{Discussion}

There have been only a few published reports on angio plus ultrasound imaging to date $(12,13)$. To the best of our knowledge, the use of angio plus imaging for parathyroid lesions has not been reported previously.

In the study, we demonstrated the superiority of angio plus imaging in terms of its ability to depict a greater number of blood vessels and detailed vascular distribution in a series of 30 parathyroid lesions. In addition, angio plus imaging was superior in revealing the polar vessel which was typical of an enlarged parathyroid, as compared with color Doppler. Vascular patterns identified by ultrasound are helpful in the diagnosis of parathyroid lesions (3,4,6-9). Generally, parathyroid adenomas are hypervascular, and a typical adenoma often has a prominent extra thyroidal feeding artery (polar vessel) entering at one pole and then extends around the periphery of the enlarged gland (6-9). The polar vessel is particularly useful in differentiating a parathyroid adenoma from a cervical lymph node, where the feeding vessel enters centrally, into the hilum (14). However, this finding is not common in all parathyroid lesions (15). In our series with color Doppler, polar vessel was detected in only $20 \%$ of parathyroid lesions. In contrast, $53.3 \%$ of them displayed polar vessel on angio plus ultrasound imaging. In this study for the first time, to our knowledge, we used angio plus imaging to detect polar vessel and detailed vascular patterns of parathyroid lesions by visualization of very low velocity flows that are not generally seen on color Doppler.

Recent studies have shown that contrast enhanced ultrasound may increase the sensitivity for detecting hyperfunctioning parathyroid glands as compared to conventional ultrasound (16-18). In addition, it allows the differentiation between parathyroid adenoma and lymph node (16-18). Parathyroid adenoma classically shows early arterial hypervascularization from the periphery to the center plus wash-out in the late phase. Lymph node shows an early enhancement of the fatty hilum, a later parenchymal enhancement without an early wash out. In our study, the sensitivity of angio plus ultrasound imaging for detecting hyperfunctioning parathyroid glands was $100 \%$, specificity could not be calculated because none of the patients had a negative histopathologic result. Our study had some limitations. First, this was a preliminary report that included a relatively small sample size of patients, and other parathyroid lesions, such as adenocarcinoma were not included. Moreover, the precise details of vessels observed with angio plus ultrasound were not validated by other imaging modalities. Moreover, this study was performed on a group of patients already selected for surgery, thus angio plus ultrasound imaging's performance in daily clinics remain unknown. In addition, the usefulness of angio plus imaging for the differential diagnosis of parathyroid lesions from lymph node or thyroid nodule was not assessed, further in-depth study is needed in future. It is expected that a combination of multi-modalities may allow a full assessment of thyroid and parathyroid lesion anatomy as well as functionality (19-24).

\section{Conclusions}

In conclusion, with angio plus ultrasound imaging, the typical increased vascularity and polar vessel commonly associated with parathyroid lesions may be obtained. Angio plus ultrasound imaging can improve the detection rate and distribution characteristics of blood flow signals of parathyroid lesions. Further research is needed to clarify the clinical meaning of increased blood flow information, such as the differentiation of parathyroid lesions from other lesions.

\section{Acknowledgments}

This work was supported by Peking Union Medical College innovative team development program.

\section{Footnote}

Conflicts of Interest: The authors have no conflicts of interest 
to declare.

Ethical Statement: The authors are accountable for all aspects of the work in ensuring that questions related to the accuracy or integrity of any part of the work are appropriately investigated and resolved. The study was approved by the institutional review board of Peking Union Medical College Hospital (No. ZS-1267) and written informed consent was obtained from all patients.

\section{References}

1. Mazzeo S, Cappelli C, Caramella D, et al. Multidetector CT in diagnostic work-up of patients with primary hyperparathyroidism. Radiol Med 2007;112:763-75.

2. Azizi G, Piper K, Keller JM, et al. Shear wave elastography and parathyroid adenoma: A new tool for diagnosing parathyroid adenomas. Eur J Radiol 2016;85:1586-93.

3. Bahl M, Muzaffar M, Vij G, et al. Prevalence of the polar vessel sign in parathyroid adenomas on the arterial phase of 4D CT. Am J Neuroradiol 2014;35:578-81.

4. Lane MJ, Desser TS, Weigel RJ, et al. Use of color and power Doppler sonography to identify feeding arteries associated with parathyroid adenomas. AJR Am J Roentgenol 1998;171:819-23.

5. Miyabe R. Three-dimensional ultrasonography before minimally invasive focused parathyroidectomy: the importance of coronal images. Surg Today 2009;39:98-103.

6. Wolf RJ, Cronan JJ, Monchik JM. Color Doppler sonography: an adjunctive technique in assessment of parathyroid adenomas. J Ultrasound Med 1994;13:303-8.

7. Varsamidis K, Vaesamidou E, Mavropoulos G. Color Doppler sonography in the detection of parathyroid adenomas. Head Neck 1999;21:648-51.

8. Scheiner JD, Dupuy DE, Monchik JM, et al. Preoperative localization of parathyroid adenomas: a comparison of power and color Doppler ultrasonogrpahy with nuclear medicine scintigraphy. Clin Radiol 2001;56:984-8.

9. Abboud B, Sleilaty G, Ayoub S, et al. Intrathyroid parathyroid adenoma in primary hyperparathyroidism: can it be predicted preoperatively? World J Surg 2007;31:817-23.

10. Kalinin AP, Pavlov AV, Alexandrov YK, et al. The Parathyroid Glands Imaging and Surgery. Berlin Heidelberg: Springer-Verlag, 2013.

11. Huppert BJ, Reading CC. The parathyroid glands. In:
Rumack CM, Wilson SR, Charboneau JW, et al. editors. Diagnostic Ultrasound 4th ed. Philadelphia (PA): Elsevier Mosby, 2011:750-71.

12. Jiang ZZ, Huang YH, Shen HL, et al. Clinical Applications of Superb Microvascular Imaging in the Liver, Breast, Thyroid, Skeletal Muscle, and Carotid Plaques. J Ultrasound Med 2019;38:2811-20.

13. Lu R, Meng Y, Zhang Y, et al. Superb microvascular imaging (SMI) compared with conventional ultrasound for evaluating thyroid nodules. BMC Med Imaging 2017;17:65.

14. Ryoo I, Suh S, You SH, et al. Usefulness of microvascular ultrasonography in differentiating metastatic lymphadenopathy from tuberculous lymphadenitis. Ultrasound Med Biol 2016;42:2189-95.

15. Acar T, Ozbek SS, Ertan Y, et al. Variable sonographic spectrum of parathyroid adenoma with a novel ultrasound finding: dual concentric echo sign. Med Ultrason 2015;17:139-46.

16. Parra Ramírez P, Hernando AS, Alcalá BB, et al. Potential utility of contrast-enhanced ultrasound in the preoperative evaluation of primary hyperparathyroidism. J Ultrasound Med 2019;38:2565-71.

17. Agha A, Hornung M, Schlitt HJ, et al. The role of contrast-enhanced ultrasonography (CEUS) in comparison with $99 \mathrm{~m}$ Technetium-sestamibi scintigraphy for localization diagnostic of primary hyperparathyroidism. Clin Hemorheol Microcirc 2014;58:515-20.

18. Zhang M, Sun L, Rui W, et al. Semi-quantitative analysis of $99 \mathrm{mTc}$-sestamibi retention level for preoperative differential diagnosis of parathyroid carcinoma. Quant Imaging Med Surg 2019;9:1394-1401.

19. Boury S. New methods for parathyroid imaging: Sonography, 4D CT, MRI. Ann Endocrinol (Paris) 2015;76:148-52.

20. Bunch PM, Kelly HR. Preoperative Imaging Techniques in Primary Hyperparathyroidism: A Review. JAMA Otolaryngol Head Neck Surg 2018;144:929-37.

21. Han Z, Lei Z, Li M, et al. Differential diagnosis value of the ultrasound gray scale ratio for papillary thyroid microcarcinomas and micronodular goiters. Quant Imaging Med Surg 2018;8:507-13.

22. Treglia G, Trimboli $P$, Huellner M, et al. Imaging in primary hyperparathyroidism: focus on the evidence-based diagnostic performance of different methods. Minerva Endocrinol 2018;43:133-43.

23. Yan F, Song Z, Du M, et al. Ultrasound molecular 
imaging for differentiation of benign and malignant tumors in patients. Quant Imaging Med Surg 2018;8:1078-83.

24. Kluijfhout WP, Pasternak JD, Gosnell JE, et al. 18F
Fluorocholine PET/MR Imaging in Patients with Primary Hyperparathyroidism and Inconclusive Conventional Imaging: A Prospective Pilot Study. Radiology 2017;284:460-7.
Cite this article as: Liu H, Liao Q, Wang Y, Hu Y, Zhu Q, Wang L, Liu Q, Li J, Jiang Y. A new tool for diagnosing parathyroid lesions: angio plus ultrasound imaging. J Thorac Dis 2019;11(11):4829-4834. doi: 10.21037/jtd.2019.11.29 